\title{
Effects of early protein undernutrition and later frontal cortex damage on habit acquisition and reversal learning in the rat
}

\author{
MARIO SILVA, ABDELAZIZ BOUZRARA, STANLEY FINGER, \\ and C. ROBERT ALMLI \\ Washington University, St. Louis, Missouri
}

\begin{abstract}
Rats from dams that were undernourished ( $8 \%$ casein diets) during pregnancy and lactation or well fed $(25 \%$ casein diets) received frontal cortical lesions at maturity. The animals were then tested for the ability to master a simple sensory discrimination and to learn reversals of that discrimination. Main effects of undernutrition and the frontal cortical lesion were found in original learning but not on the reversals, and animals that received both early undernutrition and the later focal brain lesion made more errors in original learning than did rats that sustained only undernutrition or only the lesion. The suggestion that early nutritional history may be one of a number of factors that can help to explain why patients with seemingly similar brain lesions do not always show identical symptoms is discussed.
\end{abstract}

Subjects who receive lesions of the same brain structures do not always exhibit identical degrees of behavioral impairment (Finger \& Stein, 1982). Several factors have now been proposed to account for this variability, which has been observed both with clinical populations and laboratory animals. For example, under some conditions it is known that age at the time of insult can affect the degree of functional sparing (Johnson \& Almli, 1978), as can the speed of growth or "momentum" of the lesion (Finger, 1978b). In addition, nonspecific environmental "enrichment" has also been shown to enhance behavioral recovery, at least on simple maze-learning tasks following cortical or hippocampal lesions (Finger, 1978a).

A variable that has only recently been explored in this context is the early nutritional history of the subject. Early undernutrition can affect several indices of brain function, including cellular growth and differentiation and synapse formation and myelination, and it has also been established that some behaviors can be affected if undernutrition is experienced during the period of rapid brain growth (Winick, 1976).

Support for this study came from NINCDS Grant NS-11002 and from Biomedical Research Support Grant Program, Division of Research Resources, to S. Finger. The authors would like to thank Peter Morgane and the Worcester Foundation for Experimental Biology (Grant NICHD-HD-06364) for providing the animals and the special diets for this study. M. Silva was supported by N.I.A. Training Grant AG 00030-07, and A. Bouzrara was supported by the Scientific Mission of Tunisia. S. Finger also has an appointment in the Neural Sciences Program at Washington University, and C. Robert Almli also has appointments with the Programs of Occupational Therapy and Neural Sciences, and in the Departments of Anatomy, Neurobiology and Preventive Medicine (Washington University Medical School, St. Louis, MO 63110). Address correspondence to $S$. Finger, Department of Psychology, Washington University, St. Louis, MO 63130.
These observations may be interpreted to suggest that early undernutrition can also affect recovery from later brain damage-a hypothesis that has now been examined in three recent studies from this laboratory, using the pups of rat dams that had experienced $50 \%$ less food than control dams during the lactation period.

The results of two of these studies have shown that early undernutrition can affect performance following a brain lesion later in life, even under conditions in which poor early diet has little or no effect on the scores of rats without focal brain lesions. In one case (Mangold, Bell, Gruenthal, \& Finger, 1981), in which rats with posterior cortical lesions were tested for the ability to make a brightness discrimination, poor early nutrition retarded recovery following the focal lesion. In the other, in which rats with hippocampal lesions were tested on a battery of DRL operant conditioning tasks, undernutrition attenuated the lesion effect (Finger \& Green, 1983). The generality of the latter finding was examined in a third study (Laughlin, Finger, \& Bell, 1983) in which rats were tested for spontaneous alternation, exploration, and spatial reversal learning. Here nutritional and hippocampal lesion effects were noted, but the two variables were not found to be additive or interactive.

The present study was conducted in the context of the diversity of the aforementioned findings, and was designed to learn more about how early nutrition might affect performance after a brain lesion later in life. This study differs from previous experiments in that the dams were placed on a low protein diet ( $8 \%$ casein) before pregnancy and were maintained on the diet through lactation. In addition, the offspring received frontal cortical lesions or sham operations at maturity, and were tested for the ability to learn a simple sensory discrimination and to make 
reversals of that discrimination, a lesion and behavioral problems not previously tested in this series of studies. It was hypothesized that frontal cortical lesion effects would be found, and that early undernutrition would potentiate these effects, much as had been found in the cortical lesion study by Mangold et al. (1981).

\section{METHOD}

\section{Subjects}

Charles River albino rats (CD) were mated for the first time at approximately 90 days of age in the animal laboratory facilities at Washington University. The dams were adapted to $8 \%$ (undernourished) or $25 \%$ (well-nourished) isocaloric casein diets (Teklad Mills) for 5 weeks before mating. The sires experienced matched diets, but for only 1 week. Once pregnant, the females were maintained on their specific diets throughout the periods of gestation and lactation.

The well-nourished (WN) and undernourished (UN) litters were culled to eight pups per litter 1 day after birth, and were randomly cross-fostered within a dietary condition. An attempt was made to have four males and four females in each litter. At 21 days of age, the pups were housed according to sex with four rats per plastic cage $(30.54 \times 35.6 \times 17.7 \mathrm{~cm})$. The offspring now were given free access to water and a standard lab diet, that is, Purina Rat Chow pellets with a protein content of $23 \%$. The rats were maintained on this diet until they were 90 days old, at which time surgery was performed.

\section{Surgery}

The rats were food and water deprived $18 \mathrm{~h}$ prior to surgery. Approximately half in each group ( $8 \%$ and $25 \%$ protein) and of each sex were anesthetized with sodium pentobarbitol $(1.2 \mathrm{ml} / \mathrm{kg})$ and given one-stage bilateral frontal cortex operations. Suction through glass pipettes was used for the frontal cortex ablations. An attempt was made to ablate only the cortex anterior to the motor zones and to spare deep-lying structures. Gelfoam was used to fill the holes left in the cranium. The wound was sutured with silk thread, and Bacitracin was applied to the wound. The remaining animals were subjected to the same anesthetic and cutting of the fascia, but experienced no drilling of the skull or lesions ("sham operations"). Animals in both the lesion (L) and sham-operated (SH) groups were enucleated at this time. Postoperatively, the rats were allowed to recover with free access to Purina Rat Chow and water for 10 days. At this point in time, the groups had the following compositions: WN $\times \mathrm{SH}=5$ males +2 females; $\mathrm{UN} \times$ $\mathrm{SH}=4$ males +2 females; WN $\times \mathrm{L}=6$ males +5 females; and $\mathrm{UN} \times \mathbf{L}=5$ males +2 females. The greater percentage of males, especially in the UN conditions, was primarily due to the fact that more males than females survived the surgery.

\section{Apparatus}

An enclosed single-unit T-maze was used to test the rats. The alleys measured $64 \times 22 \times 14 \mathrm{~cm}$, and the startbox was $36 \times$ $22 \times 14 \mathrm{~cm}$. On one floor of the two arms of the T-maze was a smooth aluminum plate, and on the other was a "rough" aluminum plate (Finger \& Frommer, 1968). The rough plate was milled with spiral cuts $(0.5 \mathrm{~mm})$ made with a flying cutter. The smooth plate was identical in height to the rough plate. A dish of food (ground Purina Rat Chow mixed with water) was placed at the end of each plate, although an interchangeable barrier prevented the animals from eating the food at one end. The apparatus was housed in a sound-attenuated room, and, to diminish olfactory cues, the plates were removed and cleaned with a Lysol solution and rinsed with water after each group completed a trial.

\section{Procedure}

Upon termination of the 10-day recovery period, the two groups of rats with lesions $(\mathrm{L} \times \mathrm{WN}, \mathrm{L} \times \mathrm{UN})$ and the two groups with sham operations (SH $\times$ WN, SH $\times$ UN) were gentled for 3 days. The rats were food deprived 1 day before they began rough-smooth discrimination testing in the maze. The sexes were tested separately, with the order of testing alternating on successive days and with half of the rats in each experimental condition initially assigned to the smooth tactile plate and the remainder assigned to the rough plate. The rats were required to go in the arm of the maze in which the assigned floor plate was located. The rats were rewarded with $30 \mathrm{sec}$ of access to food when the correct response was made. An incorrect response resulted in no reward. However, during the first 1-2 days of testing, rats with incorrect responses were allowed to move to the opposite arm and sample the food in order to make them aware of its availability at the end of both arsm of the maze. The location of the floor plates and the order in which the rats were run were randomly determined. The rats were allowed $10 \mathrm{~min}$ of free access to wet chow at the completion of the day's testing. They received no additional food until the next testing session but had free access to water in the home cage.

The rats received five trials per day, and the criterion for learning the tactile discrimination was a perfect score on 2 successive days. Upon reaching criterion, each rat was required to change plate preference in order to receive food, and this was followed by two more "reversals" using the same criterion as before. A period of $\mathbf{4 0}$ days without reaching criterion was used to terminate testing if criterion could not be met on a problem. These rats received a conservative maximum score of $\mathbf{4 2}$ days to criterion for statistical treatment of their data. The rats were sacrificed after the third reversal or if removed from testing for not meeting criterion within $\mathbf{4 0}$ days.

\section{Histology}

At the conclusion of testing, each rat was injected with an overdose of sodium pentobarbitol and perfused through the aorta with $0.9 \%$ saline solution followed by $10 \%$ Formalin. The brain was immediately removed and the olfactory bulbs anterior to the frontal cortex were cut. A second coronal cut was made between the occipital cortex and the cerebellum, and a third coronal cut was made just behind the cerebellum at the level of the medulla. The cerebral cortex was measured, and the block containing cerebrum and anterior brainstem was weighed. The cerebellar block, which included the pons and some medulla, also was weighed. The cerebral hemispheres were then photographed, and frozen sections were cut at $40 \mu$ on a freezing microtome. Every 10th section was saved and stained with cresyl-violet acetate for histological examination.

\section{RESULTS}

\section{Body Weights}

At the time of birth, the mean body weights for the underfed and well-fed male rat pups included in the behavioral analyses (see below) were 5.64 and $6.12 \mathrm{~g}$, respectively. For the females, the corresponding weights were 5.32 and $5.68 \mathrm{~g}$. The difference between the two groups of males used in this study was shown by $t$ tests to be significant $(p<.01)$, but, perhaps in part because there were 19 males and only 8 females, the difference between the well-fed and underfed female pups was not statistically significant $(p>.05)$.

The rats were weighed again at the time of surgery, and the difference among the males remained signif- 
icant ( 347 vs. $401 \mathrm{~g}$; t test, $p<.025$ ). The body weights of the undernourished and well-fed females were now 221 and $218 \mathrm{~g}$, respectively.

\section{Behavioral Measures}

One female WN $\times \mathrm{L}$ rat and one male UN $\times \mathrm{L}$ rat died during testing and were not included in any of the statistics. In addition, two of the female rats with lesions (WN $\times \mathrm{L}$ and $\mathrm{UN} \times \mathrm{L}$ ) showed evidence of infection or the spread of damage well beyond the boundaries of the frontal association areas. These rats also were excluded from the weight and behavioral data analyses.

Because the scores of the male and female rats within a treatment condition were directly comparable, this variable was collapsed for statistical treatment of the data. This also was done for the factor of whether the rats were first rewarded on the rough or on the smooth tactile plate, inasmuch as this factor had no effect on performance.

The data for original learning of the tactile discrimination are summarized in Figure 1. As can be seen, errors to criterion were elevated by both the brain lesion and undernutrition, with the poorest scores belonging to those rats who experienced both early undernutrition and frontal cortex ablations. A two-way ANOVA (nutrition $\times$ lesion) on these data revealed a highly significant effect of nutrition, reflecting the poorer scores of the underfed animals $[F(1,23)=9.66, p<.01]$, and a significant effect of the lesion due to more errors by the frontal cortex group $[F(1,23)=5.73, p<.05]$. The lesion $\times$ nutrition interaction effect was not significant $[F(1,23)=$ $0.62, \mathrm{p}>.05]$.

The reversal learning scores of the four groups are presented in Figure 2. The number of errors on the reversal series was highest for the group experiencing both lesion and undernutrition, and lowest for the

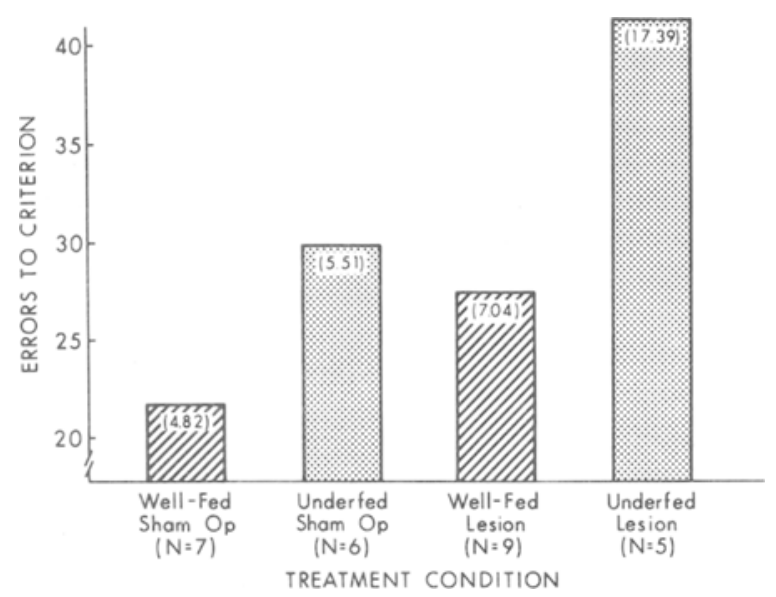

Figure 1. Performance scores of the four groups of rats in original learning of the tactile discrimination. The number in brackets at the top of each bar is the standard deviation for the group.

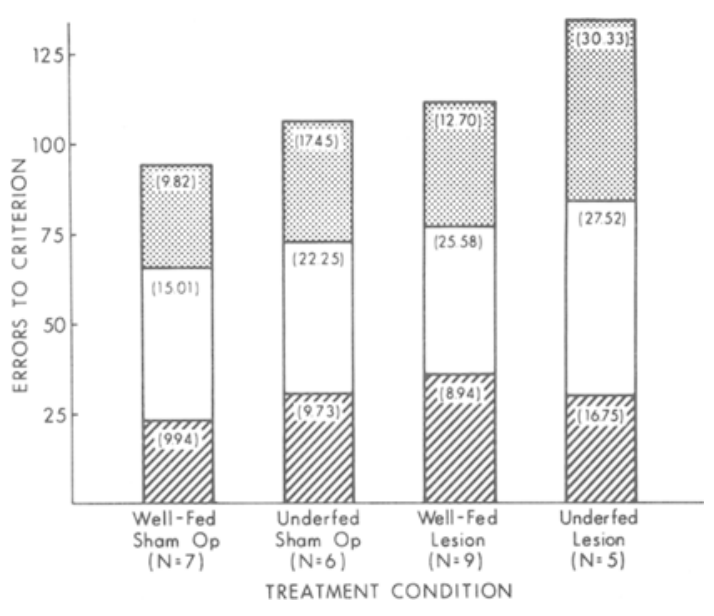

Figure 2. Performance scores of the four groups of rats on three reversals of the tactile discrimination. The number in brackets is the standard deviation for the group.

well-fed sham-operated group. The standard deviations on the reversals, however, were very high, and a three-way repeated measures ANOVA for two between factors (lesion and nutrition) and one within factor (reversals 1, 2, and 3) did not reveal significant differences for nutrition $[F(1,23)=1.25, p>.05]$ or the frontal cortex lesion $[\mathrm{F}(1,23)=2.22, \mathrm{p}>.05]$. There was a task effect $[F(2,46)=6.28, p<.01]$ due to worse performance on Reversal 2 , but no significant interaction effects. All but two rats (both undernourished $\times$ lesion) completed all of the reversals within the allotted time.

Both for original and reversal learning, the results for days to criterion were essentially the same as those for errors to criterion.

\section{Lesions}

The brains of the animals with lesions that were included in the study are presented in Figure 3. In general, there was severe damage to the dorsolateral and ventrolateral aspects of the prefrontal cortex. The lesions extended to the rhinal fissure at the rostral border, but sometimes ended about $1 \mathrm{~mm}$ above the rhinal fissure at the caudal border. The rats differed to a greater extent in the amount of damage sustained near the midsagittal sinus (anteromedial or dorsomedial frontal cortex), with some rats showing little if any tissue sparing here and others displaying lesions sparing a little over $1 \mathrm{~mm}$ of tissue on each side of the sinus. The lesions did not involve the olfactory bulbs, nor did they extend caudally into the primary motorsensory cortical area (Ms-l). In terms of subcortical involvement, the lesions did infringe upon the forceps major and the genu of the corpus callosum in many of the rats, and in five cases (three male and two female) there was bilateral involvement of the most anterior part of the caudate putamen complex. 


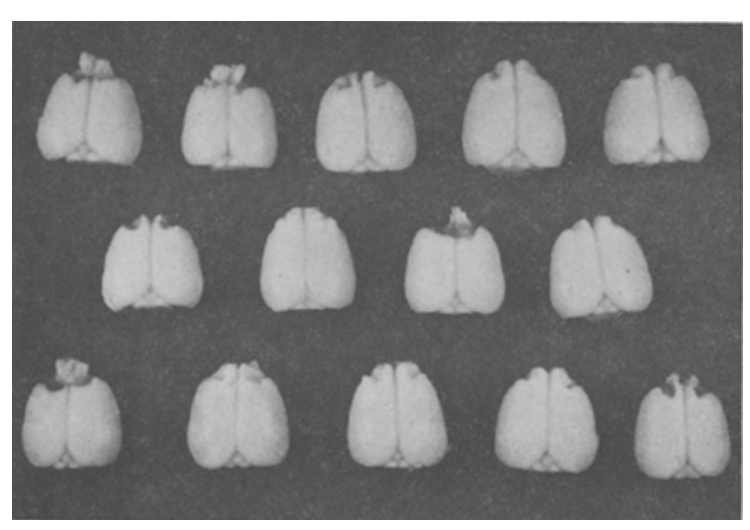

Figure 3. Dorsal view of brains of rats with frontal cortical lesions. Those in Rows 1 and 2 are from the well-fed rats, and those in Row 3 are from the underfed rats.

No differences could be found between the placements of the lesions of the well-fed and the underfed rats. Both groups showed variability in the boundaries of the lesions, especially in the anteromedial region. In addition, no feature of the damage could be found to account for the very poor scores of some of the rats in original learning or on the reversals and the less deviant or control-like scores of others. In particular, the degree of anteromedial involvement did not appear to be correlated with performance scores in original learning (or on the reversals), nor did overall lesion size. However, the two females with caudate putamen involvement made more errors than the three females in the comparable nutritional groups without bilateral caudate-putamen damage on the original tactile discrimination. Nevertheless, this possible association, based on very few animals, failed to hold for the males in original learning or for either sex on the reversal learning tasks.

\section{Brain Measures}

Because of the small number of female subjects in each condition and the possible brain size differences due to gender, only the male brains were compared. Undernutrition significantly affected the weights of the cerebral blocks in the sham-operated rats. The WN $\times$ SH animals had heavier cerebral blocks than the UN $\times$ SH rats $(t$ test, $p<.025)$. In addition, undernutrition resulted in lower cerebellar block weights [two-way ANOVA, $F(1,15)=5.32$, $\mathrm{p}<.05]$. Neither cerebral length nor width were affected by undernutrition in the sham-operated groups (t tests, ps > .05). Means and standard deviations for these measures appear in Table 1.

\section{DISCUSSION}

The main findings of the present experiment were that early undernutrition significantly impaired acquisition of a simple tactile discrimination and that prefrontal cortex damage also elevated error scores. Rats who experienced both undernutrition and a frontal cortical lesion made the most errors in original learning. However, these effects did not persist when the rats were tested on a series of reversals of the tactile habit they had just acquired.

The fact that early undernutrition impaired acquisition of the tactile discrimination is consistent with a wealth of recent studies showing that feeding $8 \%$ protein diets to female rats during pregnancy and lactation can have protracted effects on many indices of brain growth and development in their offspring. This is because cell division in the rat begins prenatally and continues into the suckling period, and because there is a marked increase in dendrites, synaptic junctions, and myelin during the first 3 postnatal weeks. As shown by Bedi, Thomas, Davies, and Dobbing (1980), Morgane et al. (1978), and Winick (1980), these and related indices of neuronal integrity can all be affected by inadequate nutrition during the "critical period" of rapid brain growth (Dobbing, 1968).

Table 1

Means and Standard Deviations of the Cerebral and Cerebellar Measures of the Four Groups of Male Rats

\begin{tabular}{|c|c|c|c|c|c|c|c|c|c|c|c|c|}
\hline \multirow[b]{4}{*}{ Measures } & \multicolumn{12}{|c|}{ Experimental Conditions } \\
\hline & \multicolumn{6}{|c|}{ Sham Operated } & \multicolumn{6}{|c|}{ Lesion } \\
\hline & \multicolumn{3}{|c|}{ Well-Fed } & \multicolumn{3}{|c|}{ Underfed } & \multicolumn{3}{|c|}{ Well-Fed } & \multicolumn{3}{|c|}{ Underfed } \\
\hline & $\mathrm{N}$ & $\mathbf{M}$ & SD & $\mathrm{N}$ & $\mathbf{M}$ & SD & $\mathbf{N}$ & $\mathrm{M}$ & SD & $\mathrm{N}$ & M & SD \\
\hline Cerebral Block Weight & 5 & 2.07 & 0.09 & 4 & 1.96 & 0.05 & 6 & $*$ & $*$ & 4 & $*$ & $*$ \\
\hline Cerebellar Block Weight & 5 & 0.68 & 0.02 & 4 & 0.62 & 0.07 & 6 & 0.65 & 0.05 & 4 & 0.61 & 0.03 \\
\hline Cerebral Length & 5 & 1.35 & 0.06 & 4 & 1.36 & 0.00 & 6 & $*$ & $*$ & 4 & $*$ & $*$ \\
\hline Cerebral Width & 5 & 1.38 & 0.05 & 4 & 1.37 & 0.03 & 6 & $*$ & $*$ & 4 & * & $*$ \\
\hline
\end{tabular}

Note-Cerebral block weight and cerebellar block weight are in grams; cerebral length and width in centimeters. cerebral lesions excluded from these analyses. 
Behavioral testing of rats reared by dams who were maintained on an $8 \%$ protein diet during pregnancy and lactation has been limited. However, Frankova and Barnes (1968) have reported that rats with inadequate protein early in life manifest a variety of behavioral disorders, including stereotyped movements and a failure to delay or extinguish fixed conditioned responses; and Picone and Hall (1977) have found that such animals also differ in their responses to tail pinch. In addition, Stern, Resnick, and Morgane (1976) reported a deficit in retention (but not in acquisition) on a response alternation task, although Hall (1983) observed no differences in learning a radial maze when factors such as running speed were equated. A review of the neurobiological and behavioral literature on the effects of $8 \%$ casein diets has been provided by Morgane et al. (1978).

The frontal cortical lesion effect that was observed in original learning is in accord with the results of experiments on rats that show that these ablations can impair a number of functions, including discrimination learning with visual and auditory stimuli (Kling, 1974, 1976). In contrast, the absence of a lesion effect on the three habit reversals may at first seem inconsistent with the results of many investigations in which rats with frontal cortical lesions were tested on reversal problems (Markowitsch \& Pritzel, 1977). Two possible explanations for the absence of a reversal learning effect merit consideration. One is that reversal learning seems to be most impaired when the medial frontal cortex is severely damaged. Although some medial frontal cortex was spared in a number of our rats, inspection of the scores of those sustaining severe damage here still failed to be suggestive of a reversal learning deficit. The second possibility is that these effects are "task specific." In support of this contention are many studies (e.g., Divac, 1971) showing reversal learning deficits with spatial cues but good performance scores with visual discriminanda (e.g., Bourke, 1954; Hannon \& Baden, 1974; see also Jeeves, 1967). The present findings would suggest that tactile stimuli may be directly comparable to visual stimuli in these experiments, although signs of a possible trend towards a lesion effect on the reversals would indicate that any proposed dissociation between roughness and spatial cues should be viewed as only tentative at this time.

The main hypothesis of the present study, that the cortical lesion effect would be enhanced by undernutrition, was confirmed on the original learning measure. Rats that experienced early undernutrition and a later frontal cortical lesion made many more errors than conspecifics that had only undernutrition or the focal brain lesion. These findings are consistent with the results obtained by Mangold et al. (1981), who found that the effects of a posterior cortical lesion on a light-dark discrimination were significantly en- hanced in rats that were undernourished early in life. However, the present experiment differs from that of Mangold et al. in several important ways. First, in this study the dietary deficiency was produced by feeding the dams $8 \%$ protein diets during pregnancy and lactation, whereas in the earlier study undernutrition was the result of a $50 \%$ reduction in food available to the dams when they were nursing. Second, the rats in the previous study received brain lesions after first learning the task and were tested for habit retention as opposed to the acquisition paradigm used here. And third, Mangold et al. observed no effect of undernutrition in the absence of a posterior cortical lesion, whereas undernutrition did affect the scores of the rats without lesions in this study.

Reports that performance after a focal cortical lesion in adulthood can be affected by undernutrition restricted to the period before weaning are important because they suggest that nutritional history is a variable that can be added to the growing list of known factors that can attenuate or potentiate the response to a brain injury. Among these factors are the speed of growth of the lesion, developmental status of the brain at the time of focal insult, environmental complexity (enrichment/deprivation), and learning history (Finger \& Stein, 1982). The fact that these conditions can affect the response to brain lesions in wellcontrolled laboratory studies would imply that the same factors may contribute to the variability in brain-damage symptomatology that is typically observed in clinical situations (Almli \& Finger, in press). Additive effects of lesion and diet, such as those found in the present experiment, and interactive effects, like those that emerged in earlier studies from this laboratory (Finger \& Green, 1983; Mangold et al., 1981), strongly suggest that a better understanding of the response to brain damage can be achieved by looking not only at the site and boundaries of the lesion, but also at the status and condition of the remaining brain-the fabric upon which the lesion is superimposed.

\section{REFERENCES}

Almui, C. R., \& Finger, S. (in press). Early brain damage: (Vol. I). Research orientations and clinical observations. New York: Academic Press.

Bedi, K. S., Thomas, Y. M., Davies, C. A., \& Dobbing, J. (1980). Synapse to neuron ratios of the frontal and cerebral cortex of 30-day-old and adult rats undernourished during early postnatal life. Journal of Comparative Neurology, 193, 49-56.

Bourke, W. T. (1954). The effects of frontal lobe damage upon habit reversal in the white rat. Journal of Comparative and Physiological Psychology, 47, 277-282.

Divac, 1. (1971). Frontal lobe system and spatial reversal in the rat. Neuropsychologia, 9, 175-183.

Doвbing, J. (1968). Vulnerable periods in developing brain. In A. N. Davison \& J. Dobbing (Eds.), Applied neurochemistry (pp. 287-316). Philadelphia: Davis.

Finger, S. (1978a). Environmental attenuation of brain lesion 
symptoms. In S. Finger (Ed.), Recovery from brain damage (pp. 297-329). New York: Plenum.

Finger, S. (1978b). Lesion momentum and behavior. In S. Finger (Ed.), Recovery from brain damage (pp. 135-164). New York: Plenum.

Finger, S., \& Frommer, G. P. (1968). Effects of cortical lesions on tactile discriminations graded in difficulty. Life Sciences, 7, 897-904.

Finger, S., \& Green, L. (1983). Early undernutrition and later hippocampal damage: I. DRL performance in rats. Nutrition and Behavior, 1, 195-206.

Finger, S., \& Stein, D. G. (1982). Brain damage and recovery. New York: Academic Press.

Frankova, S., \& Barnes, R. H. (1968). Effect of malnutrition in early life on avoidance conditioning and behavior of adult rats. Journal of Nutrition, 96, 485-493.

HALl, R. D. (1983). Is hippocampal function in the adult rat impaired by early protein or protein-caloric deficiencies? Developmental Psychobiology, 16, 395-411.

HANNon, R., \& BADEN, A. (1974). A comparison of frontal pole, anterior median and caudate nucleus lesions in the rat. Physiology \& Behavior, 13, 513-521.

JeEves, M. A. (1967). Some paradoxical effects of bilateral lesions in the frontal cortex in rats. Neuropsychologia, 5, 73-84.

Johnson, D., \& Almli, C. R. (1978). Age, brain damage and performance. In S. Finger (Ed.), Recovery from brain damage (pp. 115-134). New York: Plenum.

KLING, J. O. (1974). Effects of frontal ablations on auditory discrimination in the rat. Journal of General Psychology, 91, 75-79.

KLING, J. O. (1976). Effects of frontal ablations upon visual pat- tern discrimination. Journal of General Psychology, 94, 303-304.

Laughlin, N. K., Finger, S., \& Bell, J. (1983). Early undernutrition and later hippocampal damage: Effects on spontaneous behaviors and reversal learning. Physiological Psychology, 11, 269-277.

Mangold, R. F., Bell, J., Gruenthal, M., \& Finger, S. (1981). Undernutrition and recovery from brain damage: $A$ preliminary investigation. Brain Research, 230, 406-411.

Markowitsch, H. J., \& Pritzel, M. (1977). Comparative analysis of prefrontal learning functions in rats, cats, and monkeys. Psychological Bulletin, 84, 817-837.

Morgane, P. J., Miller, M., Kemper, T., Stern, W., Forbes, W., Hall, R., Bronzino, J., Kissane, J., Hawrylewicz, E., \& RESNICK, O. (1978). The effects of protein malnutrition on the developing central nervous system in the rat. Neuroscience and Biobehavioral Reviews, 2, 137-230.

Picone, T. A., \& Hall, R. D. (1977). Effects of early protein malnutrition on tail-pinch behavior of the rat. Bulletin of the Psychonomic Society, 10, 269. (Abstract)

Stern, W. C., Resnick, O., And Morgane, P. J. (1976). Developmental protein malnutrition in rats: Acquisition and longterm retention of a single alternation discrimination. Nutritional Reports International, 14, 475-483.

Wintck, M. (1976). Malnutrition and brain development. New York: Oxford University Press.

WiNick, M. (1980). Nutrition and central nervous system development. Progress in Brain Research, 53, 93-97.

(Manuscript received October 23, 1983; revision accepted for publication March 16, 1984.) 